\title{
Foreword
}

\section{Recognition of the Mediterranean diet: going a step further}

\author{
Lluís Serra-Majem ${ }^{1,2, *}$, Anna Bach ${ }^{1}$ and Blanca Roman ${ }^{1}$ \\ ${ }^{1}$ Foundation for the Advancement of the Mediterranean Diet, Barcelona Science Parc, C/Baldiri Reixac 4, \\ 08028 Barcelona, Spain: ${ }^{2}$ Department of Clinical Science, University of Las Palmas de Gran Canaria, Spain
}

The Mediterranean diet has developed and progressed extensively since the end of the last century. The leadership of the Foundation for the Advancement of Mediterranean Diet (FDDM) has potentially contributed to this exponential growth of evidence and research and also increased public awareness. However, there is a very small number of systematic reviews analysing the effect of the Mediterranean diet on health-related issues, and the number of randomised controlled clinical trials is also low (fewer than 50). In contrast, the high-level worldwide popularity of the Mediterranean diet as a healthy and recommended diet is evidenced by the proliferation of media attention and population awareness (more than 740000 citations in Google ${ }^{\circledR}$, January 2005) ${ }^{1}$.

Most of the scientific articles published are observational epidemiological studies (primarily ecological or casecontrol studies and a few cohorts). Almost all the reviews published are non-systematic and reflect an opinion or a collection of self-selected articles rather than an objective analysis of sound evidence ${ }^{1}$. Also, difficulties in reproducing epidemiological data in different settings have been identified $^{2,3}$ due to several reasons, mostly related to the lack of understanding that the composition and characteristics of the Mediterranean diet are more than the sum of a few foods groups, as interpreted in certain non-Mediterranean countries ${ }^{4}$. Thus, most of the studies analyse the effect of a Mediterranean-type diet where olive oil has been substituted by other fats or where some components from other regions have been added to the traditional Mediterranean diet. This kind of study usually does not provide any improvement in the evidence about the Mediterranean diet, but rather has a detrimental effect ${ }^{5}$.

With this collection of papers presented at the Fifth Barcelona International Congress on the Mediterranean Diet and published in this issue of Public Health Nutrition, the FDDM aims to pool and increase evidence-based nutrition and health within the context of the traditional Mediterranean diet (Fig. 1).

Mediterranean diet recommendations need to be evidence-based, which implies further development of clinical and observational nutritional epidemiology in Mediterranean countries. Also, objective systematic (nonpersonalised) reviews need to address different areas of the relationship between the Mediterranean diet and health ${ }^{1}$. Research should emphasise the comparison of
Mediterranean food patterns with globalised Western food patterns that have an increasing impact on Mediterranean countries. In particular, conflicts of interest may arise as research on specific food groups or patterns financed by related food industries or institutions may influence its consumption and, therefore, have important economic consequences. In this way, the research on the Mediterranean diet may need to be particularly independent and unambiguous. Consequently, the FDDM calls on the European Commission and national research bodies to support research in this area apart from the financing already provided by private food companies and other institutions. Otherwise, the promotion of the Mediterranean diet will always have shortcomings and thus continue to be viewed with certain misgivings.

On the decennial of the Barcelona Declaration, in 2005 the Year of the Mediterranean, after the Fifth Barcelona International Congress on the Mediterranean Diet, a meeting at the University of Rome La Sapienza was organised as the Third Euro-Mediterranean Forum 'Dialogues between civilizations and people: The food cultures'. Participants represented national and regional agencies, universities, cultural institutions and individual experts in various food, nutrition and cultural fields, coming from all over the Mediterranean region. Among the conclusions of the Forum we have selected five key points ${ }^{6}$ :

- To remember that the ancient Greek word 'diaita' means equilibrium and lifestyle; therefore, the traditional Mediterranean Diet is more than just a diet, it is a whole lifestyle pattern with physical activity playing an important role.

- To take into account the intense scientific activity over the last few decades that has significantly contributed to understanding the relationship between nutrition and health. The health benefits of the dietary traditions of the Mediterranean populations have contributed to the acceptance of the Mediterranean diet as a healthy dietary model, that can help in the prevention of noncommunicable diseases and obesity.

- To establish as a priority a cohesive definition of the traditional Mediterranean diet (so as to be equivalent to Mediterranean food or food culture) in order for all Mediterranean countries to present a shared perspective 


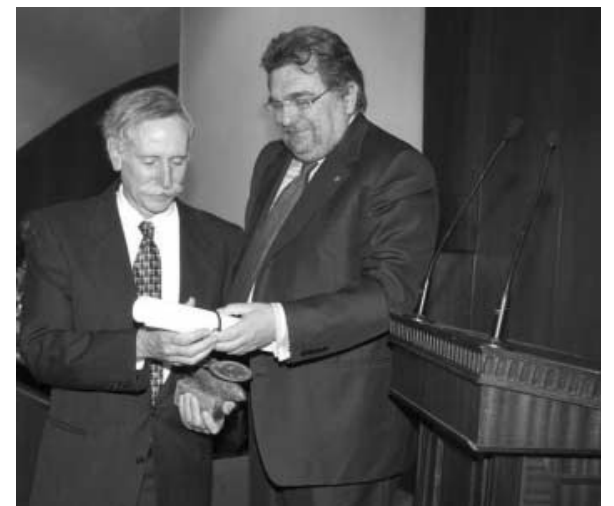

Fig. 1 The Catalan Minister of Agriculture, Mr Antoni Siurana, gives the $\mathrm{V}$ Grande-Covian Award of the FDDM to Professor Walter Willett, in recognition of his research contribution and career, during the Fifth Barcelona International Congress on the Mediterranean Diet

and strategy. The definition should refer to the traditional Mediterranean diet as a means of preserving cultural heritage. Main foods included in the common food basket are: an abundance of olive oil and olives, fruits, vegetables, cereals (mostly unrefined), legumes, nuts and fish, moderate amounts of dairy products (preferably cheese and yoghurt) and low quantities of meat and meat products. Wine in moderation is acceptable when it is not contradictory to religious or social norms. But the idiosyncrasy of the pattern is not only a list of foods (some traditional) but that it also refers to sustainability (mostly fresh, seasonal and locally grown products) as well as preparation techniques following traditional recipes and the way and context of eating them, which are also key components of the Mediterranean diet. It is emphasised that the Mediterranean diet is complete and usually does not need any kind of supplement or enrichment unless recommended for health reasons and for a few nutrients (folic acid and iodine).

- To take into account that the traditional Mediterranean diet has health implications besides cultural and economic implications. Therefore, all Mediterranean countries need to agree and contribute to the process of preservation and promotion. To start the process, obtaining United Nations Educational, Scientific and Cultural Organization (UNESCO) backing for the recognition of the Mediterranean diet as a part of Food Cultural Heritage is an initial and shared common objective to be coordinated from the Barcelona counterpart as an extension of the 1995 Barcelona Declaration, in collaboration with all the Mediterranean country representatives.

\section{References}

1 Serra-Majem L, Roman B, Estruch R. Scientific evidence of interventions using the Mediterranean diet: a systematic review. Nutrition Reviews 2006; 64(2): 531-52.

2 Esposito K, Marfella R, Ciotola M, Di Palo C, Giugliano F, Giugliano $G$, et al. Effect of a Mediterranean-style diet on endothelial dysfunction and markers of vascular inflammation in the metabolic syndrome: a randomized trial. JAMA 2004; 292(12): 1440-6.

3 Michalsen A, Lehmann N, Pithan C, Knoblauch NT, Moebus S, Kannenberg F, et al. Mediterranean diet has no effect on markers of inflammation and metabolic risk factors in patients with coronary artery disease. European Journal of Clinical Nutrition 2005 Nov 23: [Epub ahead of print].

4 Serra Majem L, Trichopoulou A, Ngo de la Cruz J, Cervera P, García-Álvarez A, La Vecchia C, et al., on behalf of the International Task Force on the Mediterranean Diet. Foreword: does the definition of the Mediterranean diet need to be updated? Public Health Nutrition 2004; 7(7): 927-9.

5 Horton R. Expression of concern: Indo-Mediterranean Diet Heart Study. Lancet 2005; 366(9483): 354-6.

6 Dernini S. Towards the advancement of the Mediterranean food cultures. Public Health Nutrition 2006; 9(1A): 103-4. 